\title{
Optically thick clumps - not the solution to the Wolf-Rayet wind momentum problem?
}

\author{
J. C. Brown ${ }^{1}$, J. P. Cassinelli ${ }^{2}$, Q. Li ${ }^{1,3}$, A. F. Kholtygin ${ }^{4,6}$, and R. Ignace ${ }^{5}$ \\ 1 Department of Physics and Astronomy, University of Glasgow, Glasgow, G12 8QQ, UK \\ e-mail: john@astro.gla.ac.uk \\ 2 Department of Astronomy, University of Wisconsin-Madison, USA \\ 3 Department of Astronomy, Beijing Normal University, PR China \\ e-mail: li@astro.gla.ac.uk \\ 4 Astronomical Institute, St. Petersburg University, Saint Petersburg State University, VV Sobolev Astronomical Institute, \\ 198504 Russia \\ 5 Department of Physics, Astronomy, \& Geology, East Tennessee State University, USA \\ ${ }^{6}$ Isaac Newton Institute of Chile, St. Petersburg Branch, Russia
}

Received 23 March 2004 / Accepted 24 June 2004

\begin{abstract}
The hot star wind momentum problem $\eta=\dot{M} v_{\infty} /(L / c) \gg 1$ is revisited, and it is shown that the conventional belief, that it can be solved by a combination of clumping of the wind and multiple scattering of photons, is not self-consistent for optically thick clumps. Clumping does reduce the mass loss rate $\dot{M}$, and hence the momentum supply, required to generate a specified radio emission measure $\varepsilon$, while multiple scattering increases the delivery of momentum from a specified stellar luminosity $L$. However, in the case of thick clumps, when combined the two effects act in opposition rather than in unison since clumping reduces multiple scattering. From basic geometric considerations, it is shown that this reduction in momentum delivery by clumping more than offsets the reduction in momentum required, for a specified $\varepsilon$. Thus the ratio of momentum deliverable to momentum required is maximal for a smooth wind and the momentum problem remains for the thick clump case. In the case of thin clumps, all of the benefit of clumping in reducing $\eta$ lies in reducing $\dot{M}$ for a given $\varepsilon$ so that extremely small filling factors $f \approx 10^{-4}$ are needed.

It is also shown that clumping affects the inference of $\dot{M}$ from radio $\varepsilon$ not only by changing the emission measure per unit mass but also by changing the radio optical depth unity radius $R_{\text {rad }}$, and hence the observed wind volume, at radio wavelengths. In fact, for free-free opacity $\propto n^{2}$, contrary to intuition, $R_{\text {rad }}$ increases with increasing clumpiness.
\end{abstract}

Key words. stars: circumstellar matter - stars: mass-loss - stars: winds, outflows - stars: Wolf-Rayet

\section{Momentum problem}

If one infers the mass loss rate $\dot{M}$ for hot massive (especially Wolf-Rayet) stars from the radio emission measure $\varepsilon=$ $\int_{V} n^{2} \mathrm{~d} V$, using a smooth spherical wind model, one finds that the wind "momentum" rate $\dot{p}=\dot{M} v_{\infty}=\eta L / c$ involves $\eta \gg 1$, where $L / c$ is the radiative momentum outflow rate (Cassinelli $\&$ Castor 1973). Insofar as such winds are believed to be radiatively driven, this poses a "momentum problem", the solution of which has long been a hot topic in the field (Barlow et al. 1981; Abbott et al. 1986; Cassinelli \& van der Hucht 1987; Willis 1991; Lucy \& Abbbot 1993; Springmann 1994; Springmann \& Puls 1995; Gayley et al. 1995; Owocki \& Gayley 1999). Estimates of $\eta$ vary according to assumptions (e.g., arguing for a high value of $L$ ) but values of $\eta$ ranging up to nearly 100 are mentioned (Hamann \& Koesterke 1998). There are two main strands of argument quite widely believed to combine to solve the momentum problem, one being mainly observationally driven and the other mainly theoretical.
The values of $\dot{M}$ associated with these large $\eta$ are those inferred from a smooth spherical wind density model, the radio emitting material filling the volume. The contribution to $\varepsilon$ from any volume element $\Delta V$ is $\Delta \varepsilon \approx n^{2} \Delta V \propto \dot{M}^{2} / \Delta V$. If, however, the material is clumpy, filling only a fraction $f=\langle n\rangle^{2} /\left\langle n^{2}\right\rangle$ of the volume, then $\Delta \varepsilon$ is enhanced by a factor $1 / f$ for a given $\dot{M}$. The mass loss rate $\dot{M}$ required to generate an observed $\varepsilon$ thus scales as $\dot{M} \propto f^{1 / 2}$ in clumpy winds. For strong clumping $(f \ll 1)$, this ameliorates the momentum problem, though the $f=10^{-4}$ required to reduce $\dot{M} v_{\infty}$ by a factor of 100 seems very unlikely, so this clumping effect alone cannot be the complete answer (e.g., Nugis \& Lamers 2000, cite clumping corrected mass-loss rates yielding $\eta \approx 6$ ). For example, making clumps very small increases their radio optical thickness and may make optically thin emission measures irrelevant. There is extensive observational evidence for large scale clumping in WR winds: the presence of narrow emission features moving out on broad wind emission lines (e.g., Robert et al. 1989, 1991; 
Moffat \& Robert 1991; Kholtygin 1995); broad band photometric and polarimetric fluctuations (e.g., Brown et al. 1995; Li et al. 2000); and the absence of strong electron scattering wings (which scales as $\langle n\rangle^{2}$ rather than $\left\langle n^{2}\right\rangle$, Hillier 1991).

On the theory side, it has long been recognised that the limit $\dot{p}=\dot{M} v_{\infty} \approx L / c$ is only true if (all) photons are scattered once only. If the wind scattering optical depth is high, the photons can, loosely speaking, be scattered "back and forth" across the wind delivering momentum of up to $2 \mathrm{hv} / \mathrm{c}$ at each scattering (for thick clumps) until $v$ is progressively dissipated by Doppler reddening at each momentum-delivering scattering on the moving matter. The nature of this multiple scattering has been described with progressively greater insight over the years. In particular, Gayley et al. (1995) showed that scattering back and forth across the entire wind is not required. Instead, the momentum is delivered in a series of random semi-local scatterings of photons as they diffuse outward, provided successive scatterings involve long enough paths to sample different matter velocities. The essential feature is that of the large scattering optical depth $\tau$, which enhances the momentum delivery rate to $\tau L / c$ (e.g., Friend \& Castor 1983; Kato \& Iben 1992; Netzer \& Elitzur 1993; Gayley et al. 1995), because the diffusive delivery scales with the number of scatterings $N_{\mathrm{s}}$ as $N_{\mathrm{s}}^{1 / 2}$, while $N_{\mathrm{s}}=\tau^{2}$. Since the predominant driver is via the large opacity/cross-section associated with lines, Gayley et al. (1995) and Owocki \& Gayley (1999) have suggested that the issue is not so much a momentum problem as an opacity problem.

The massive WR winds are still believed to be driven by line opacity (e.g., Lucy \& Abbott 1993). Unlike the less massive winds of OB stars, the WR winds have significant ionization gradients, that can substantially alter the line opacity distribution with radius in the flow (Herald et al. 2000; Vink et al. 2000). Consequently, as the photons move away from the star, interact with a certain line opacity that exists at some radius $r$ in the flow, and then escape, the photons encounter a new line opacity distribution at a different radius. Consequently, if there are gaps in the line distribution at one radius, those gaps can be filled by a different line distribution that exists in another part of the wind flow. The opacity problem then represents how effectively all of these gaps are "filled".

Photon escape at gaps in the line frequency forest reduces the flux mean opacity (or flux mean cross section $\sigma$ per particle in our formulation) used in the gray approximation. The maximum that can be achieved by multiple scattering is reached when the number of random scatterings is so great as to Doppler shift the photons down to near zero frequency, the maximum Doppler shift per scattering being of order $v / c$ for wind speed $v$. This requires $\tau \approx N_{s}^{1 / 2} \approx c / v(\approx 100)$, implying $\dot{M} v_{\infty} \approx L / c \times c / v_{\infty}$ or $\dot{M} v_{\infty}^{2} / 2 \approx L / 2$ which is the energy conservation limit. Available calculations of multiple scattering with real opacities can yield $\eta$ gains of order 10 , that may explain some WR winds, but not the more extreme cases in which $\eta \sim 10^{2}$ is required.

Since reduction of $\dot{M}$ (for a given $\varepsilon$ ) by clumping and increase of momentum delivery by multiple scattering can each offer a factor of order 10 reduction in the momentum problem, there seems to be growing widespread belief that the momentum problem can be laid to rest (e.g., Conti 1995).
However, this involves the tacit assumption that these two factors can operate independently and constructively, the impact of clumping on the effectiveness of multiple scattering never having been addressed (Hillier \& Miller 1999; although Shaviv (1998) has discussed the related topic of how optically thick clumps increase the Eddington luminosity for novae). Here we show, using simple geometric arguments, that this assumption is incorrect in the case of optically thick clumps, and that clumping, while reducing $\dot{M}$, also reduces $\tau$, so making multiple scattering less effective. Essentially this is because clumping reduces the number of scattering centres compared to scattering off of atoms and also reduces $\langle n\rangle$, for a given $\varepsilon$. (Note that when discussing the effects of clumping it is essential to keep in mind that the observed $\varepsilon$ is held fixed. This fact is sometimes overlooked.)

We find quantitatively that, for thick clumps, the reduction in multiple scattering momentum delivery more than offsets the reduction in momentum required, the nett effect being that clumping worsens the momentum problem rather than solving it.

\section{Single clump}

To illustrate the point, we first consider one thick scattering clump of mass $M$ composed of atoms/ions of mass $m$. This is taken to have very high internal optical depth in the line-driving wavelength range so the clump as a whole is the scattering centre. Since we are not concerned with the wind speed profile $v(r)$ but only with the final wind speed and momentum, we here approximate clumps as moving radially with speed $v \approx v_{\infty}$ and to have the shape of a conical slice of radial thickness $\delta$ and solid angle $\Omega$, the volume of the cone being $r^{2} \Omega \delta$ at distance $r$. We assume the clump to be optically thin at radio wavelengths, so its radio flux depends on the emission measure, but optically thick to lines for the stellar radiation at short wavelengths that are responsible for driving the flow.

The emission measure $\varepsilon_{1}=n^{2} V$ (which measures the radio emission rate) of a single clump is

$\varepsilon_{1}=\frac{(M / m)^{2}}{r^{2} \Omega \delta}=\frac{\varepsilon_{1 \mathrm{o}}}{x^{2}}$

where $\varepsilon_{1 \mathrm{o}}=(M / m)^{2} /\left(R_{\mathrm{rad}}^{2} \Omega \delta\right)$ the clump emission measure at $r=x R_{\text {rad }}=R_{\text {rad }}$, for $R_{\text {rad }}$ the radius of the radio photosphere which may be hundreds of times larger than the optical photosphere radius. Note that, for a prescribed $\varepsilon_{1}, M \propto \sqrt{\Omega}$ for any chosen $r, \delta$.

On the other hand, the available rate of delivery of momentum is

$\dot{p}_{1, \text { avail }}=\frac{L}{c} \frac{\Omega}{4 \pi}$

where we ignore scale factors of order unity due to the effects of gravity and of the backward scattering angular distribution function. The rate of momentum delivery required is

$\dot{p}_{1, \text { req }} \approx \frac{M v}{r / v}$. 
It follows that, for a given $\varepsilon_{1}$, the effectiveness of momentum delivery to a single clump is

$\Psi_{1}=\frac{\dot{p}_{1, \text { avail }}}{\dot{p}_{1, \text { req }}}=\left[\frac{L}{4 \pi m v^{2} c \varepsilon_{1}^{1 / 2}}\right]\left(\frac{\Omega}{\delta}\right)^{1 / 2}$.

This decreases as we make $\Omega$ smaller - i.e., as we make the clump clumpier - because the momentum $\dot{p}_{1 \text {,req }}$ required $\propto M \propto \sqrt{\Omega}$, but the momentum $\dot{p}_{1 \text {,avail }}$ available $\propto \Omega$, and the decline of the latter with $\Omega$ is dominant for a single clump. That is, making the $\Omega$ of a single clump smaller does reduce $\dot{p}_{1 \text {,req }}$ for a given $\varepsilon_{1}$ but reduces $\dot{p}_{1 \text {,avail }}$ even more. So shrinking one clump of a given $\varepsilon_{1}$ makes it harder to drive it to terminal speed of known value $v_{\infty}$.

Compressing the clump radially does help (in this single thick clump case) since reducing $\delta$ reduces $M v$ for prescribed $\varepsilon_{1}, \Omega$.

\section{Multiple clumps}

We now have to consider the effect of multiple scattering in the case of a multiple clump wind, since multiple scattering cannot occur in the case of an individual discrete clump. In doing so we take all clumps to be optically thick in the UV but thin in the radio, identical in size and mass, and use the gray opacity approximation, the clumps being driven by a spectral mean "continuum" radiation flux. We are of course well aware that in reality there will be a distribution of clump sizes and masses. However, if one can prove that for any specific clump parameters, clumping reduces the benefit of multiple scattering, then the same must be true of the sum over any distribution of clump parameters so long as they remain thick. Put another way, the arguments that clumping a wind increases its emission measure, that multiple scattering increase momentum delivery, and that clumping reduces multiple scattering all derive essentially from geometric arguments and have nothing to do with the details of opacity or of clump size distribution (other than being thick).

Retention of the conical slice shape described above, taking $\Omega$ and $\delta$ independent of $r$, means that the clumps expand in 2-D (transversely) rather than in 3-D, which is reasonable for a highly supersonic wind. The constant $\Omega, \delta$ assumption also means that, for constant $v$, clumps occupy the same fraction (constant filling factor $f$ ) of the volume at all $r$. For spherical (3-D) clump expansion, linear radial expansion $(\delta \propto r)$ would result, for constant $v$, in radial merging of clumps, which corresponds to an $r$-dependent filling factor $f$ with $f \rightarrow 1$ as clumps merge. Situations with non-constant filling factor $f=f(r)$ have been discussed by Nugis, Crowther, \& Willis (1998); Hillier \& Miller (1999); and Ignace et al. (2003). We assume clumps are, on average, emitted uniformly over the stellar surface at a rate $\dot{C}$ in clumps per second. Then the space density of clumps at $r$ is

$\mathcal{N}(r)=\frac{\dot{C}}{4 \pi r^{2} v}$,

where we again approximate $v=$ constant $=v_{\infty}$ and the radio emission measure of one clump is again given by Eq. (1). Using
Eqs. (1) and (5), the total emission measure can be written

$\varepsilon=\int_{R_{\mathrm{rad}}}^{\infty} 4 \pi r^{2} \mathrm{~d} r \mathcal{N} \varepsilon_{1}=\frac{\dot{C}}{R_{\mathrm{rad}} v}\left(\frac{M}{m}\right)^{2} \frac{1}{\Omega \delta}=\frac{\dot{C} R_{\mathrm{rad}}}{v} \varepsilon_{1 \mathrm{o}}=N_{\mathrm{eff}} \varepsilon_{1 \mathrm{o}}$.

The last form is interesting, showing that the total emission measure $\varepsilon$ is just the initial emission measure of one clump $\varepsilon_{1 \mathrm{o}}$ at $r=R_{\text {rad }}$ times an effective number of clumps $N_{\text {eff }}=\dot{C} R_{\text {rad }} / v$, namely that located in the range $R_{\text {rad }} \leq r<2 R_{\text {rad }}$.

The mass loss rate $\dot{M}$ and the momentum delivery rate $\dot{p}_{\text {req }}$ required are

$\dot{M}=\dot{C} M$

and

$\dot{p}_{\text {req }}=\dot{C} M v$,

where we neglect scale factors of order unity as we did in Eq. (2). By Eqs. (6) and (8), we get the momentum delivery rate required for a given total wind emission measure $\varepsilon$ as a function of $M, \Omega$, namely

$\dot{p}_{\text {req }}=m^{2} v^{2} R_{\text {rad }} \varepsilon \frac{\Omega \delta}{M}$.

We want to compare this with the momentum delivery rate available from multiple scattering of stellar photons and we take this to be given by (cf. Sect. 1)

$\dot{p}_{\text {avail }}=\frac{L}{c} N_{\mathrm{s}}^{1 / 2}=\frac{L}{c} \tau$

where $\tau$ is the mean (gray approximation) line scattering optical depth of the wind and $N_{\mathrm{s}}=\tau^{2}$ is the number of scatterings of an escaping photon. $\tau$ is also the "covering factor" or the total solid angle of all the clumps as seen from the star divided by $4 \pi-$ see Appendix.

The wind optical depth for starlight due to lines treated in the gray approximation is (for individually thick clumps)

$\tau=\int_{R_{\mathrm{UV}}}^{\infty} n Q \mathrm{~d} r$

where $n$ is the density of scatterers, $Q$ is the cross section of scatterers, and $R_{\mathrm{UV}}$ is the UV photosphere associated with the dominant line driving.

We choose to split the range into two sectors, $r<d$ and $r>d$, where $d$ is the distance at which an individual clump becomes optically thin radially. At $r<d$ the individual scatterer is a clump of area $r^{2} \Omega$ and thickness $\delta$, while at $r>d$, it is an ion of area $\sigma$ (the actual value adopted for $\sigma$ being some frequency average over lines). Thus the optical depth integral expands to

$$
\begin{aligned}
\tau & =\int_{R_{\mathrm{UV}}}^{\mathrm{d}} \mathcal{N}(r) r^{2} \Omega \mathrm{d} r+\int_{d}^{\infty} \mathcal{N}(r) \frac{M}{m} \sigma \mathrm{d} r \\
& =\frac{\dot{C} R_{\mathrm{UV}}}{4 \pi v}\left[\Omega\left(\frac{d}{R_{\mathrm{UV}}}-1\right)+\frac{M \sigma}{m R_{\mathrm{UV}}^{2}} \frac{R_{\mathrm{UV}}}{d}\right]
\end{aligned}
$$

where $d$ satisfies

$\frac{M \sigma}{m} \frac{1}{\Omega d^{2}}=1$ 
and thus

$\frac{d}{R_{\mathrm{UV}}}=\left(\frac{M \sigma}{m R_{\mathrm{UV}}^{2}}\right)^{1 / 2} \frac{1}{\Omega^{1 / 2}}$.

Then Eq. (12) becomes

$$
\begin{aligned}
\tau & =\frac{\dot{C} R_{\mathrm{UV}}}{4 \pi v}\left[2\left(\frac{M \sigma}{m R_{\mathrm{UV}}^{2}}\right)^{1 / 2} \Omega^{1 / 2}-\Omega\right] \\
& =\frac{\dot{C}}{4 \pi v}\left(\frac{M \sigma}{m}\right)^{1 / 2} \Omega^{1 / 2}\left[2-\left(\frac{m R_{\mathrm{UV}}^{2}}{M \sigma}\right)^{1 / 2} \Omega^{1 / 2}\right] .
\end{aligned}
$$

Consider the second term in expression (15). The ratio $\frac{M \sigma}{m}$ is the total area of all the atoms in a clump, and $R_{\mathrm{UV}}^{2} \Omega$ is the total area of a clump at $r=R_{\mathrm{UV}}$. Since our analysis deals with individually thick clumps, we require $\frac{M \sigma}{m} \gg R_{\mathrm{UV}}^{2} \Omega$, so we get $d \gg R_{\mathrm{UV}}$ and can neglect the second term in Eq. (15) to write

$\tau=\frac{\dot{C}}{2 \pi v}\left(\frac{M \sigma}{m}\right)^{1 / 2} \Omega^{1 / 2}$.

If we express $\dot{C}$ in terms of $\varepsilon$ by using Eq. (6), then Eq. (16) becomes

$\tau=\frac{R_{\mathrm{rad}} \sigma^{1 / 2}}{2 \pi}\left(\frac{m}{M}\right)^{3 / 2} \varepsilon \delta \Omega^{3 / 2}$.

Now we get the available momentum using Eqs. (10) and (17) in terms of $M, \Omega$ for a given $\varepsilon$, namely

$\dot{p}_{\text {avail }}=\frac{L}{c}\left(\frac{R_{\mathrm{rad}} \sigma^{1 / 2}}{2 \pi}\right)\left(\frac{m}{M}\right)^{3 / 2} \varepsilon \delta \Omega^{3 / 2}$.

Comparing Eqs. (9) and (18), we find a dimensionless measure of the effectiveness of momentum delivery, as the ratio of momentum available to momentum required, as a function of $M$, $\Omega$, for a given $\varepsilon$, namely

$\Psi=\frac{\dot{p}_{\text {avail }}}{\dot{p}_{\text {req }}}=\left[\frac{L}{2 \pi v^{2} c R_{\text {rad }}}\left(\frac{\sigma}{m}\right)^{1 / 2}\right]\left(\frac{\Omega R_{\text {rad }}^{2}}{M}\right)^{1 / 2}$.

For a given star $(L)$ and a wind of given speed $v$ and composition $(m, \sigma)$, the expression in the bracket [ ] of Eq. (19) is constant, so we can vary the value of $\Psi$ by varying the clump parameter combination $\Omega / M$.

The essential result is that $\Psi$ increases with increasing $\Omega / M$, i.e., with increasing clump cross section per unit mass (which is different from the single clump case of Eq. (4)). To minimise the momentum problem (maximise $\Psi$ ) for a given mass $M$ (and thickness $\delta$ ), $\Omega$ should be as large as possible while for a given $\Omega$ the mass $M$ should be as low as possible with, in both cases, $\dot{C}$ varying according to Eq. (6) to ensure the correct $\varepsilon$. If we change (e.g., increase) $\delta, \Psi$ does not change but $\dot{C}$ changes (falls) to maintain fixed $\varepsilon$. Consequently, to maximise $\Psi$ we must make the clump mass small, the clump angle large, and the clump thickness large with correspondingly small $\dot{C}$, all of these corresponding to minimising clumping.

It is also of interest to express $\Psi$ in terms of the volume filling factor $f=\langle n\rangle^{2} /\left\langle n^{2}\right\rangle$ which can be expressed (with $V_{\mathrm{c}}=$ single clump volume $=\Omega r^{2} \delta$ at $r$ ) as

$f=\frac{4 \pi r^{2} \mathrm{~d} r \mathcal{N}(r) V_{\mathrm{c}}}{4 \pi r^{2} \mathrm{~d} r}=\frac{\dot{C} R_{\mathrm{rad}}}{v} \frac{\Omega}{4 \pi} \frac{\delta}{R_{\mathrm{rad}}}=N_{\mathrm{eff}} f_{\Omega} f_{\mathrm{r}}$ where $f_{\Omega}=\frac{\Omega}{4 \pi}$ and $f_{\mathrm{r}}=\frac{\delta}{R_{\mathrm{rad}}}$ are solid angle and radial filling factors respectively. Alternatively, the volume filling factor can be expressed as

$f=\frac{R_{\mathrm{rad}} \varepsilon m^{2}}{4 \pi}\left(\frac{\Omega \delta}{M}\right)^{2}$.

Comparing Eqs. (19) and (21), we see that, under our thick clump assumption, $\Omega / M \propto f^{1 / 2}$ so that $\Psi \propto f^{1 / 4}$, so that for any values of $(\varepsilon, \delta)$, decreasing $f$ decreases the effectiveness of momentum delivery.

All of the above shows that, contrary to conventional "wisdom", in the case of thick clumps, clumping does not help solve the momentum problem but actually makes it worse.

The case of a smooth wind can be considered a limit of the clumpy case as the clumps blend. However, there are infinitely many clumpy cases that approach the smooth case as the clumps blend and it is easier to evaluate $\Psi=\Psi_{\mathrm{o}}$ for the smooth case directly using $n_{\mathrm{o}}(r)=\dot{M} /\left(4 \pi r^{2} m v\right)$. Then with subscript "o" denoting the smooth case, we get

$\dot{p}_{\text {o,req }}=\dot{M} v$,

$\varepsilon_{\mathrm{o}}=\frac{\dot{M}^{2}}{m^{2}} \frac{1}{4 \pi v^{2} R_{\mathrm{rad}}}$,

$\tau_{\mathrm{o}}=\frac{\dot{M} \sigma}{4 \pi m v R_{\mathrm{UV}}}$

and

$\dot{p}_{\text {o,avail }}=\frac{L}{c} \tau_{\mathrm{o}}=\frac{L}{c} \frac{\dot{M} \sigma}{4 \pi m v R_{\mathrm{UV}}}$

from which we deduce that

$\Psi_{\mathrm{o}}=\frac{L}{c} \frac{1}{4 \pi v^{2} R_{\mathrm{UV}}} \frac{\sigma}{m}$.

Explicitly comparing the momentum delivery effectiveness for the clumpy and smooth cases we have, by Eqs. (19) and (26)

$\frac{\Psi}{\Psi_{\mathrm{o}}}=2\left[\left(\frac{\Omega R_{\mathrm{UV}}^{2}}{M}\right) /\left(\frac{\sigma}{m}\right)\right]^{1 / 2}=\left(\frac{\left(2 R_{\mathrm{UV}}\right)^{2}}{\frac{M \sigma}{m \Omega}}\right)^{1 / 2}=\frac{2 R_{\mathrm{UV}}}{d}$

which is clearly $\ll 1$ for clumps which are initially optically thick. Note also that $\Psi$ involves the ratio of $\Omega\left(2 R_{\mathrm{UV}}\right)^{2} / M$ and $\sigma / m$, respectively, the cross-sections per unit mass of clumps and of atoms, while $\Psi_{\mathrm{o}}$ involves only $\sigma / \mathrm{m}$. Clearly, the continuous limit corresponds to $\Omega\left(2 R_{\mathrm{UV}}\right)^{2} / M \rightarrow \sigma / m$ at $d \rightarrow 2 R_{\mathrm{UV}}$ (cf. Eq. (13)), the "clumps" become individual atoms, becoming thick at that point. Note that $d \rightarrow R_{\mathrm{UV}}$ is essentially the limit where driving approaches the smooth wind limit, equally applicable to optically thin clumps.

Carrying this line of inquiry further, it is helpful to see how Eq. (19) for $\Psi$ approaches the smooth limit $\Psi_{0}$. We require that $\tau=\tau_{\mathrm{o}}$ from Eqs. (16) and (24), that $\varepsilon=\varepsilon_{\mathrm{o}}$ from Eqs. (6) and (23), and finally that $f=1$. These conditions are met for

$\frac{\dot{C} R_{\mathrm{rad}}}{v} \frac{\Omega}{4 \pi} \frac{\delta}{R_{\mathrm{rad}}}=N_{\mathrm{eff}} f_{\mathrm{r}} f_{\Omega}=1$,

$\frac{M \sigma}{m}=\Omega\left(2 R_{\mathrm{UV}}\right)^{2}$ 
and

$\delta=\frac{16 \pi m v R_{\mathrm{UV}}^{2}}{\dot{M} \sigma}$

which, on substitution in Eq. (19) gives $\Psi=\Psi_{\text {o }}$ (Eq. (26)) as required. To interpret Eq. (29) physically, note that $M \sigma / m$ is the total cross sectional area of all the atoms in one clump while $\Omega\left(2 R_{\mathrm{UV}}\right)^{2}$ is the cross sectional area of one clump at $r=2 R_{\mathrm{UV}}$. These can only be equal if clumps have the scale of individual atoms. Secondly, Eq. (30) can be expressed as $4 \pi\left(2 R_{\mathrm{UV}}\right)^{2}=$ $\frac{\dot{M} \sigma \delta}{m v}$. Here $\frac{\dot{M}_{\sigma}}{m v}$ is the total area of all clump atoms per unit radial distance, so $\frac{\dot{M}_{\sigma \delta}}{m v}$ is the total area of all clump atoms in a scale length $\delta$. Thus, since $4 \pi\left(2 R_{\mathrm{UV}}\right)^{2}$ is the spherical area at $r=2 R_{\mathrm{UV}}$, the scale $\delta$ defines the range of $r$ around $2 R_{\mathrm{UV}}$ in a smooth wind over which the clump atoms just cover the sphere.

\section{Discussion and conclusions}

\subsection{Conclusions regarding thick clumps}

Using a simple model we have shown that while clumping reduces the mass loss rate of WR stars required by radio emission measures, it also reduces the wind optical depth and hence multiple scattering and momentum delivery. The nett result is that thick clumping worsens the momentum discrepancy rather than solving it. This is not the case for thin clumps, as we discuss below.

\subsection{Discussion of assumptions}

First we comment on various simplifications we have made which might modify our results somewhat. These include the approximation of constant $v$, relaxation of which does not seem very likely to change our results much since the radio emission measure is produced well out in the winds beyond where wind acceleration starts. Secondly, in common with many authors, we have so far assumed that the inner boundary $R_{\text {rad }}$ (and hence the volume) of the radio emission measure region does not change with clumping. In fact, one might expect, by analogy with the UV optical depth (Eq. (17)), that the radio optical depth might fall with increased clumping, reducing $R_{\text {rad }}$ and increasing the radio source volume and emission measure. To check this, we first want to know the radio optical depth $\tau^{\prime}$ for a clumped wind. This is roughly given by

$\tau^{\prime}(r)=\int_{r}^{\infty} \mathcal{N}(r) \frac{M}{m} \sigma^{\prime} \mathrm{d} r$

where $\sigma^{\prime}$ is the relevant cross section per proton. However, we have to note that the main radio absorption mechanism is freefree opacity which is density dependent $\left(\sigma^{\prime} \propto n_{\mathrm{c}} \approx 1 / r^{2}\right)$ and we have to write

$\sigma^{\prime}=\sigma_{\mathrm{o}}^{\prime} \frac{n_{\mathrm{c}}(r)}{n_{\mathrm{o}}}$

where $n_{\mathrm{c}}=n_{\mathrm{o}}$ and $\sigma^{\prime}=\sigma_{\mathrm{o}}^{\prime}$ are defined in any reference level $r=r_{\mathrm{o}}$. Then Eq. (31) becomes

$\tau^{\prime}(r)=\frac{\dot{C}}{12 \pi v} \frac{\sigma_{\mathrm{o}}^{\prime}}{n_{\mathrm{o}}}\left(\frac{M}{m}\right)^{2} \frac{1}{\Omega \delta r^{3}}$, so that now $R_{\text {rad }}$ is given by $\tau^{\prime}\left(R_{\text {rad }}\right)=1$, namely

$$
R_{\mathrm{rad}}=\left(\frac{\dot{M} \sigma_{\mathrm{o}}^{\prime}}{12 \pi v n_{\mathrm{o}} m^{2}}\right)^{1 / 3}\left(\frac{M}{\Omega \delta}\right)^{1 / 3} .
$$

Consequently, increasing $M$ for given $\Omega, \delta$ or decreasing $\Omega, \delta$ for a given $M$ (i.e., increasing clumpiness) actually makes $R_{\text {rad }}$ bigger, not smaller, in a clumpy wind because of the density dependence of free-free absorption.

The corresponding emission measure expression is now as before but based on the new clumping dependent value in Eq. (34) of $R_{\text {rad }}$ which leads to

$\varepsilon=\frac{3^{1 / 3}}{(4 \pi)^{2 / 3}}\left(\frac{\dot{M}}{v}\right)^{2 / 3}\left(\frac{n_{\mathrm{o}}}{m^{4} \sigma_{\mathrm{o}}^{\prime}}\right)^{1 / 3}\left(\frac{M}{\Omega \delta}\right)^{2 / 3}$

which does increase as we increase $M / \Omega \delta$ (i.e., clumpiness) for a given $\dot{M}$ but now with $\varepsilon \propto[\dot{M} M /(\Omega \delta)]^{2 / 3}$, instead of $[\dot{M} M /(\Omega \delta)]$ for the constant $R_{\text {rad }}$ case. Thus although clumpiness still reduces $\dot{M}$ for a given $\varepsilon$, it does so less than with constant $R_{\mathrm{rad}}$ and likewise, thick clumps are now even less helpful to the momentum problem.

\subsection{Discussion of thin clumps}

Throughout, we have considered here the case when each clump is an optically thick scatterer at line-driven wavelengths. The other case of relevance to reducing $\eta$ is where there is strong clumping $(f \ll 1)$ but with very large numbers of very small scale clumps, each optically thin. We have considered here the case when each clump is an optically thick scatterer at line driving wavelengths but with a large overall wind optical depth. In this case our analysis does not really apply and the driving has to be described by multiple scatterings over the small Sobolev optical depths of many successive individual thin scatterers. The nett effect here is to yield the same momentum delivery rate as in a smooth wind but to reduce $\dot{M}$ for a given $\varepsilon$ by increasing the optically thin radio emission measure $\varepsilon_{1}$ of each clump. In this thin clump limit, clumping does not reduce momentum delivery by photon escape, as happens for thick clumps, but reduces $\dot{M}$ for a given $\varepsilon$. As a solution to the momentum problem, this scenario puts the entire onus on the reduction of $\dot{M} / \varepsilon$ and of $\eta$ by a factor $f^{1 / 2}$ and for the extreme case of $\eta \approx 100$ it requires $f \approx 10^{-4}$. How such a huge compression of wind matter into clumps is achievable physically, and whether it can be done without making the clumps optically thick in the radio (bearing in mind that the radio opacity $\propto n^{2}$ ) are questions that must be addressed before clumping solutions of the momentum problem, are to be accepted.

\subsection{Other solutions to the momentum problem?}

Since clumping solutions, thick or thin, remain questionable, we briefly mention here some other possibly relevant factors. Some have advocated non-spherical models for the massive WR flows. Lamers \& Pauldrach (1991) developed a bistability model for early-type stars (e.g., B[e] stars), and Poe et al. (1989) proposed a two-component model with fast winds from polar regions and a slow flow from equatorial regions, 
later termed the Luminous Magnetic Rotator model (Cassinelli 1991). In aspherical models such as these, the high radio flux arises from the denser equatorial region, whereas the high terminal speeds derive from a line-of-sight that lies perhaps in a broad polar region. These two-component structures have not been widely accepted because few WR stars show substantial (non-varying) intrinsic polarizations, as would be expected for stars that have a dense equatorial flow (Harries et al. 1998). However, Taylor \& Cassinelli (1992) studied the cancellation of polarization owing to a more tenuous polar wind, and it was surprisingly effective. Whether or not the solution to the momentum problem lies in the aspherical structure of such a rotational model, or yet some other aspherical picture, our paper has shown that the wide spread belief that the solution lies in the clumpy + multiple scattering picture is too simplistic.

Acknowledgements. The authors acknowledge support for this work from: a NATO Collaboration Grant (A.F.K., J.C.B., J.P.C.); a UK PPARC Research Grant (J.C.B.); NASA Grant Number TM4-5001X (J.P.C., J.C.B.); Royal Society Sino-British Fellowship Trust Award (Q.L.); a NSFC grant 10273002 (Q.L.); and a RFBR grant 01-0216858 (A.F.K.). We thank the referee (Ken Gayley) whose comments led to a significant improvement of the paper.

\section{Appendix A: Relation between Covering factor $Y$, number of scatterings $N_{\mathrm{S}}$, and optical depth $\tau$}

$Y$ is the fraction of the solid angle around a star that is covered by scatterers. Let $A$ and $\omega$ be the cross section and solid angle for one scatterer at $r$, so that $\omega=A(r) / r^{2}$ and let $\mathcal{N}_{\mathrm{s}}(r)$ be the space density of scatterers, then the covering factor at $r$ is the total solid angle of all the scatterers divided by $4 \pi$, namely

$$
\begin{aligned}
Y=\frac{1}{4 \pi} \int_{r}^{\infty} \mathcal{N}_{\mathrm{s}}(r) \omega 4 \pi r^{2} \mathrm{~d} r & =\frac{1}{4 \pi} \int_{r}^{\infty} \mathcal{N}_{\mathrm{s}}(r) \frac{A(r)}{r^{2}} 4 \pi r^{2} \mathrm{~d} r \\
& =\int_{r}^{\infty} \mathcal{N}_{\mathrm{s}}(r) A(r) \mathrm{d} r=\tau(r) .
\end{aligned}
$$

A photon travelling in a medium with typical size $D$, density $\mathcal{N}_{\mathrm{s}}$, and particle cross section $A$, on average undergoes $N_{\mathrm{s}}$ scatterings before escaping. Its mean free path $l=1 /\left(\mathcal{N}_{\mathrm{s}} A\right)$, is related to $N_{\mathrm{s}}$ by $l \sqrt{N_{\mathrm{s}}}=D$, so

$N_{\mathrm{s}}=\left(\frac{D}{l}\right)^{2}=\left(\mathcal{N}_{\mathrm{s}} A D\right)^{2}=\tau^{2}$

\section{References}

Abbott, D. C., Torres, A. V., Bieging, J. H., \& Churchwell, E. 1986, ApJ, 303, 239

Barlow, M. J., Smith, L. J., \& Willis, A. J., 1981, MNRAS, 196, 101

Brown, J. C., Richardson, L. L., Antokhin, I., et al. 1995, A\&A 295, 725

Cassinelli, J. P., \& Castor, J. I. 1973, ApJ, 179, 189

Cassinelli, J. P., \& van der Hucht, K. A., 1987, in Instabilities in Luminous Early Type Stars, ed. H. J. G. Lamers, \& C. W. H. De Loore, Astrophysics and Space Science Library (Dordrecht: Reidel), 136, 231
Cassinelli, J. P. 1991, in Wolf-Rayet Stars and Interrelations with Other Massive Stars in Galaxies, ed. K. A. van der Hucht, \& B. Hidayat (Dordrecht: Kluwer), Proc. IAU Symp., 143, 289

Conti, P. S. 1995, in Wolf-Rayet Stars: Binaries; Colliding Winds; Evolution, ed. K. A. van der Hucht, \& P. M. Williams (Dordrecht: Kluwer), Proc. IAU Symp., 163, 565

Friend, D. B., \& Castor, J. I. 1983, ApJ, 272, 259

Gayley, K. G., Owocki, S. P., \& Cranmer, 1995, ApJ, 442, 296

Hamann, W. R., \& Koesterke, L. 1998, A\&A, 333, 251

Harries, T. J., Hillier, D. J., \& Howarth, I. D., 1998, MNRAS, 296, 1072

Herald, J. E., Schulte-Ladbeck, R. E., Eenens, P. R. J., \& Morris, P. 2000, ApJS, 126, 469

Hillier, D. I. 1991, A\&A, 247, 455

Hillier, D. I., \& Miller, D. L. 1999, ApJ, 519, 354

van der Hucht, K. A. 1992, A\&AR, 4, 123

Ignace, R., Oskinova, L. M., \& Foullon, C. 2000, MNRAS, 318, 214

Ignace, R., Quigley, M. F., \& Cassinelli, J. P. 2003, ApJ, 596, 538

Kato, M., \& Iben, I. 1992, ApJ, 394, 305

Kholtigin, A. F. 1995, in Wolf-Rayet Stars: Binaries; Colliding Winds, ed. K. A. van der Hucht, \& P. M. Williams (Dordrecht: Kluwer), Evolution: Proc. IAU Symp., 163, 160

Lamers, H. J. G. L. M., \& Pauldrach, A. W. A., 1991, A\&A, 244 , L5

Li, Q., Brown, J. C., Ignace, R., Cassinelli, J. P., \& Oskinova, L. M. 2000, A\&A 357, 233

Lucy, L. B., \& Abbott, D. C. 1993, ApJ, 405, 738

Moffat, A. F. J., \& Robert, C. 1991, in Wolf-Rayet Stars and Interrelations with Other Massive Stars in Galaxies, ed. K. A. van der Hucht, \& B. Hidayat (Dordrecht: Kluwer), Proc. IAU Symp., 143, 109

Netzer, N., \& Elitzur, M. 1993, ApJ, 410, 701

Nugis, T., Crowther, P. A., \& Willis, A. J. 1998, A\&A, 333, 956

Nugis, T., \& Lamers, H. J. G. L. M. 1993, ApJ, 410, 701

Owocki, S. P., \& Gayley, K. G. 1995, in Wolf-Rayet Stars: Binaries; Colliding Winds; Evolution, ed. K. A. van der Hucht, \& P. M. Williams (Dordrecht: Kluwer), Proc. IAU Symp., 163,138

Owocki, S. P., \& Gayley, K. G. 1999, in Wolf-Rayet Phenomena in Massive Stars and Starburst Galaxies, ed. K. A. van der Hucht, G. Koenigsberger, \& P. R. J. Eenens (San Francisco, Calif: ASP), Proc. IAU Symp. 193, 157

Poe, C. H., Friend, D. B., \& Cassinelli, J. P. 1989, ApJ, 337, 888

Robert, C., Moffat, A. F. J., Bastien, P., Drissen, L., \& St.-Louis, N. 1989, ApJ, 347, 1034

Robert, C., Moffat, A. F. J., \& Seggewiss, W., 1991, in Wolf-Rayet Stars and Interrelations with Other Massive Stars in Galaxies, ed. K. A. van der Hucht, \& B. Hidayat (Dordrecht: Kluwer), Proc. IAU Symp., 143, 147

Shaviv, N. J. 1998, ApJ, 494, L193

Springmann, U. 1994, A\&A, 289, 505

Springmann, U., \& Puls, J. 1995, in Wolf-Rayet Stars: Binaries; Colliding Winds; Evolution, ed. K. A. van der Hucht, \& P. M. Williams (Dordrecht: Kluwer), Proc. IAU Symp., 163, 170

Vink, J. S., de Koter, A., \& Lamers, H. J. G. L. M., 2000, A\&A, 362, 295

Willis, A. J. 1991, in Wolf-Rayet Stars and Interrelations with Other Massive Stars in Galaxies, ed. K. A. van der Hucht \& B. Hidayat (Dordrecht: Kluwer), Proc. IAU Symp., 143, 265 